\title{
First case of AML with rare chromosome translocations: a case report of twins
}

\author{
Lin Wang ${ }^{1}$, Yanhua Sun ${ }^{2}$, Yanli Sun ${ }^{3}$, Lingbin Meng ${ }^{4}$ and $X i n ~ X u^{5,6^{*}}$
}

\begin{abstract}
Background: Leukemia is different from solid tumor by harboring genetic rearrangements that predict prognosis and guide treatment strategy. PML-RARA, RUNX1-RUNX1T1, and KMT2A-rearrangement are common genetic rearrangements that drive the development of acute myeloid leukemia (AML). By contrast, rare genetic rearrangements may also contribute to leukemogenesis but are less summarized.

Case presentation: Here we reported rare fusion genes ZNF717-ZNF37A, ZNF273-DGKA, and ZDHHC2-TTTY15 in a 47year-old AML-M4 patient with FLT3 internal tandem duplication (ITD) discovered by whole genome sequencing (WGS) using the patient's healthy sibling as a sequencing control.

Conclusion: This is, to our knowledge, the first case of AML with fusion gene ZNF717-ZNF37A, ZNF273-DGKA, and ZDHHC2-TTTY15.
\end{abstract}

Keywords: AML, Genetic rearrangement, FLT3/ITD, WGS

\section{Background}

Chromosome translocations are common genetic abnormality in leukemia. Multiple genetic fusions have been summarized and can be used to predict prognosis and for targeting. For example, in The 2016 revision of the World Health Organization (WHO) classification of myeloid neoplasms and acute leukemia, AML with recurrent genetic abnormalities were classified into AML with $\mathrm{t}(8 ; 21)(\mathrm{q} 22 ; \mathrm{q} 22.1) ; R U N X 1-R U N X 1 T 1$, AML with inv. (16)(p13.1q22) or t(16;16)(p13.1;q22);CBFB-MYH11, APL with PML-RARA, AML with $\mathrm{t}(9 ; 11)(\mathrm{p} 21.3 ; \mathrm{q} 23.3) ; M L L T 3-$ KMT2A, AML with $\mathrm{t}(6 ; 9)(\mathrm{p} 23 ; \mathrm{q} 34.1) ; D E K-N U P 214$, AML with inv.(3)(q21.3q26.2) or t(3;3)(q21.3;q26.2); GATA2, MECOM, AML (megakaryoblastic) with $\mathrm{t}(1 ; 22)$ (p13.3; 13 . 3);RBM15-MKL1 [1]. These AML subtypes account for most AML with recurrent genetic abnormalities. However, rare genetic translocations exist. For example, in spite of the fact that six partners of $K M T 2 A$ gene account for majority KMT2A-rearranged leukemia, more than 135 partners of $K M T 2 A$ have been identified so far [2]. Rare

\footnotetext{
* Correspondence: xinxu@wfmc.edu.cn

${ }^{5}$ Stem Cell Lab of the Affiliated Hospital of Weifang Medical University, Weifang 261053, Shandong, China

${ }^{6}$ College of Bioscience and Technology, Weifang Medical University, \#1 Building Room 610, 288 Shenglidong Street, Weifang, Shandong Province 261042, People's Republic of China

Full list of author information is available at the end of the article
}

translocations may also contribute to leukemogenesis and be useful for personalized medicine of leukemia.

Thanks to the development of next generation sequencing, variations with low population frequency are able to be identified. The identification of susceptible or driver genes are also accelerated by twin or family study. Here we reported rare fusion genes ZNF717-ZNF37A, ZNF273$D G K A$, and ZDHHC2-TTTY15 in AML with FLT3/ITD by a twin study.

\section{Case presentation \\ Patient's history, clinical and molecular features}

A 47-year-old male (T3) presented to hospital at 21 June, 2015 reporting fever and being hypodynamic. Routine blood test showed high leukocyte count (115. $\left.27 \times 10^{9} / \mathrm{L}\right)$, anemia $(\mathrm{Hb} 84 \mathrm{~g} / \mathrm{L})$, a total platelet count of $259 \times 10^{9} / \mathrm{L}$, and C-reactive protein (CRP) of $40.9 \mathrm{mg} / \mathrm{L}$. Primitive and immature cells made up $90 \%$ of the peripheral blood cells. Acute leukemia was diagnosed and the patient was hospitalized. Bone marrow aspiration showed that primitive and immature cells made up 97\% of the bone marrow cells and an AML-M4 was diagnosed. Immunophenotyping showed full expression of HLA-DR and CD33, partial expression of CD7, CD117, CD13, CD34, CD38, CD25, FMC-7, CD56, CD64, $\mathrm{CD} 11 \mathrm{C}$ and MPO, no expression of CD5, CD10, CD19, 
CD20, CD14, CD103, CD23, CD41a, GlyA, CD11b, CD15, CD138, kappa, lambda, CD79a, TdT, and cCD3. Chromosome karyotype of bone marrow showed 46, XY [3]. FLT3/ITD was identified. WT1/ABL ratio was 133. $74 \%$. Pirarubicin+cytarabine were administered but bone marrow depression occurred. Bone marrow aspiration showed active myeloproliferative activity and primitive and immature cells made up 54\% of the bone marrow cells. The patient left hospital.

The patient presented to hospital second time at 24 July, 2015. Routine blood test showed leukocyte count of 18 . $70 \times 10^{9} / \mathrm{L}, \mathrm{Hb}$ of $69 \mathrm{~g} / \mathrm{L}$, platelet count of $30 \times 10^{9} / \mathrm{L}$. Bone marrow aspiration showed primitive and immature cells made up $79 \%$ of the bone marrow cells. IEA (Idarubicin + Etoposide + Aza-C) regimen was administrated but bone marrow depression occurred. Bone marrow aspiration showed active myeloproliferative activity and primitive and immature cells made up $92 \%$ of the bone marrow cells. The patient left hospital.

The patient presented to hospital third time at 17 August, 2015. Routine blood test showed leukocyte count of $61.46 \times 10^{9} / \mathrm{L}, \mathrm{Hb}$ of $62 \mathrm{~g} / \mathrm{L}$, platelet count of $101 \times 10^{9} / \mathrm{L}$. Homoharringtonine+Etoposide were administrated. The patient left the hospital at 19 October, 2015.

The patient presented to hospital fourth time at 1 November, 2015. Routine blood test showed leukocyte count of $145.72 \times 10^{9} / \mathrm{L}, \mathrm{Hb}$ of $71 \mathrm{~g} / \mathrm{L}$, platelet count of $15 \times 10^{9} / \mathrm{L}$. The patient deteriorated rapidly and passed away.

The patient had a twin brother (T4) who is healthy. Short tandem repeat (STR) genotyping based on 21 loci (D19S433, D5S818, D21S11, D18S51, D6S1043, AMEL, D3S1358, D13S317, D7S820, D16S539, CSF1PO, Penta D, D2S441, vWA, D8S1179, TPOX, Penta E, TH01, D12S391, D2S1338, FGA) identified T3 and T4 were monozygotic twins.

\section{Whole genome sequencing}

Peripheral blood mononuclear cells (PBMCs) from the patient T3 (leukocyte count of $145.72 \times 10^{9} / \mathrm{L}$ ) and healthy sibling T4 were collected and genome DNA were isolated for WGS. Paired end $150 \mathrm{bp}$ (PE150) sequencing on Illumina HiSeq $\mathrm{X}$ was performed at the Core Genomic Facility of Beijing Annoroad Genomics. All data were aligned to hg19 with BWA, arranged with samtools, marked with Picard, locally aligned with GATK. The coverage rate at $30 \times$ is $89.82 \%$.

Single nucleotide polymorphism (SNP) was annotated using ANNOVAR. A total 3,033,876 SNPs were shared between T3 and T4. T4 had 317,907 unique SNPs while T3 had 301,334 unique SNPs.

Insertion-deletion (Indel) was annotated using ANNOVAR. A total 515,603 InDels were shared between T3 and T4. T4 had 93,904 unique SNPs while T3 had 92,676 unique SNPs.

Structural variation (SV) was annotated using ANNOVAR. A total 444 SVs were shared between T3 and T4. T4 had 152 unique SVs while T3 had 136 unique SVs. Only 23 (3.97\%) SVs are exonic and only 4 relevant genetic loci (AKR1C4, CLCNKB/FAM131C, NUP43, PPIAL4D/E/F) are unique for T3.

Copy number variation (CNV) was annotated using ANNOVAR. A total 316 CNVs were shared between T3 and T4. T4 had 35 unique CNVs while T3 had 48 unique CNVs (21 are exonic).

There are 3 unique fusion pairs in $\mathrm{T} 3$ which are ZNF717 (exon6) fused to ZNF37A (exon 8), ZNF273 (exon 15) fused to DGKA (exon 10), and $Z D H H C 2$ (exon 1) fused to TTTY15 (exon 13) as shown in Fig. 1 and specified in Additional file 1.

\section{Discussion and conclusions}

Here, we reported the first case of AML-M4 in a 47 years old man bearing ZNF717-ZNF37A, ZNF273-DGKA, and $Z D H H C 2-T T T Y 15$ fusions detected by WGS analysis.

ZNF717 has been reported to be involved in hepatocellular carcinoma [4], gastric cancer [5], cervical cancer [6]. From TCGA fusion gene database (www.tumorfusions.org), ZNF717-FOXP1 fusion was found in one colon adenocarcinoma. From another fusion gene database (Dong lab's database, http://donglab.ecnu.edu.cn/ databases/FusionCancer/), ZNF717 was found to be fused with $L O C 100132288$ and ITGB1, respectively, in lung cancer.

ZNF273 was reported by the database (http://donglab. ecnu.edu.cn/databases/FusionCancer/) to be fused with $T P R K B$ in two cases, one in melanoma and another in prostate cancer.

$D G K A$, the gene encoding diacylglycerol kinase alpha (DGK $\alpha)$ which is a negative regulator of oncogene Ras [7], has attracted much interests from cancer researchers recently due to its involvement in multiple signaling pathways. DGK $\alpha$ inhibition compromises cancer cell viability, impairs angiogenesis, and notably boost $\mathrm{T}$ cell activation and enhance cancer immunotherapies [8]. $D G K A$ was reported to form fusions with $A S B 8$ in prostate cancer and $R A B 5 B$ in uterine carcinosarcoma by TCGA database. DGKA was also reported to be fused with STARD4 in 4 Burkitt's lymphoma cases and with $C D 74$ in one lung cancer from Dong lab's database.

$D G K A$ may play a role in leukemogenesis. DGK $\alpha$ was absent in non-differentiated human promyelocytic leukemia cell line HL-60 cells, but was robustly upregulated during differentiation. By contrast, the other DGK isoforms $(\delta, \varepsilon, \gamma$, $\zeta)$ existed in undifferentiated HL-60 cells but were remarkably decreased throughout differentiation [9]. DGK $\alpha$ was also reported to be abundant in the nuclei of human 


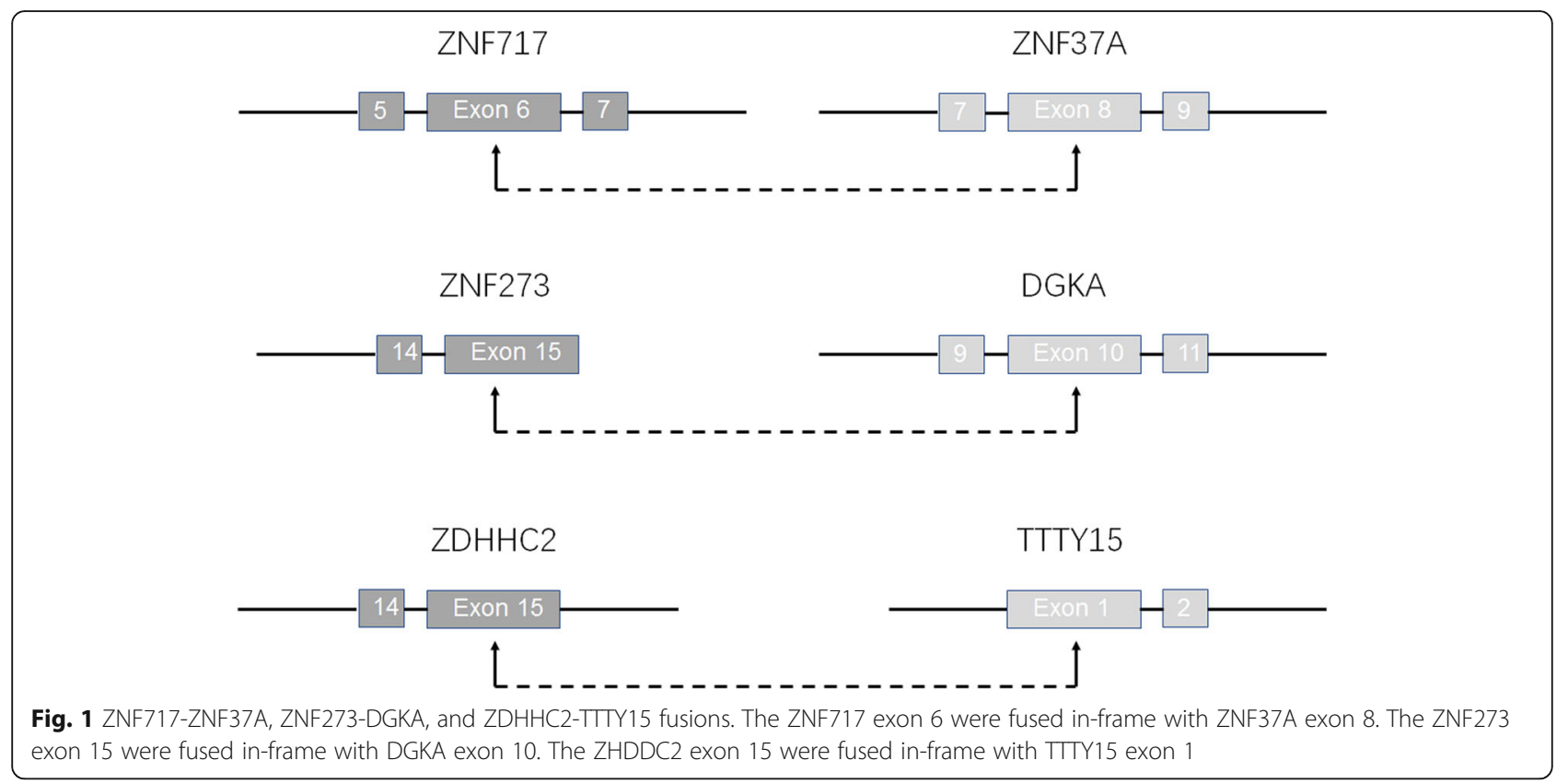

erythroleukemia cell line K562, and to be involved in cell cycle progression of $K 562$ cells [10]. The information implicates that DGK $\alpha$ may be involved in the differentiation and cell cycle progression of leukemia cells.

The fusion between ZNF273 and DGK $\alpha$ may result in the production of a new protein with changed localization that may in turn influence how the kinase activity of DGK $\alpha$ exerts. The fusion between ZNF273 and DGK $\alpha$ led to the replacement of $\mathrm{N}$-terminal domain of DGK $\alpha$ by the whole Zn finger domain of ZNF273 (Fig. 2). Deletion of the N-terminal domain of DGK $\alpha$ was reported to confer no effect on enzyme activity but result in constitutive localization of DGK $\alpha$ at the plasma membrane in intact $\mathrm{T}$ cells [11]. ZNF273-DGK $\alpha$ fusion may lead to dysregulated signaling pathway in leukemia cells.

$Z D H H C 2$, a palmitoyl acyltransferase [12], has been reported by multiple groups to be involved in gastric adenocarcinoma [13], hepatocellular carcinoma [14]. $Z D H H C 2$ was reported to be fused with $L T B P 1$ in two breast cancer cases, with $P P P 2 R 2 A$ in one ovarian cancer, with FGD6 in one sarcoma case from TCGA database.

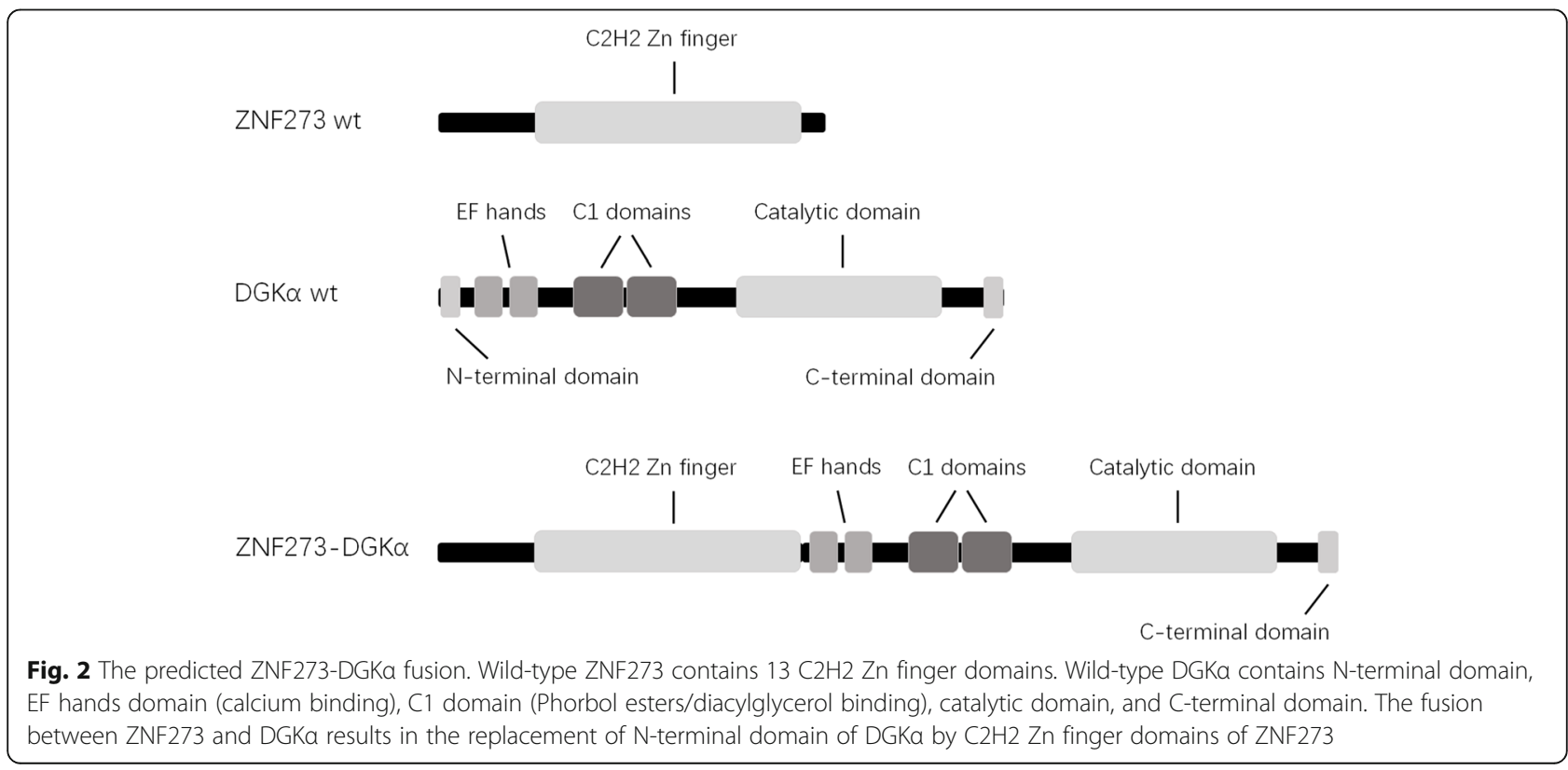


TTTY15 fusions have been reported in prostate cancer [3]. TTTY15-USP9Y fusion has been found in multiple cases of hepatocellular cancer, lung cancer, melanoma, prostate cancer, implicating a potential driver function of TTTY15-USP9Y fusion in carcinogenesis.

To sum up, ZNF717-ZNF37A, ZNF273-DGKA, and $Z D H H C 2-T T T Y 15$ fusions may contribute to the development of AML.

\section{Additional file}

Additional file 1: Fusion Report. (XLS $2 \mathrm{~kb})$

\section{Abbreviations}

AML: Acute myeloid leukemia; CNVs: Copy number variations; InDel: Insert-deletion; ITD: Internal tandem duplication; SNPs: Single nucleotide polymorphisms; SVs: Structural variations; WGS: Whole genome sequencing

\section{Acknowledgements}

The authors would like to thank Shizhe Liu, Juan Wang from Annoroad Genomics who gave suggestions about WGS.

\section{Funding}

The research was supported by the Scientific Research Foundation for the Returned Overseas Chinese Scholars State Education Ministry, Shandong Provincial Natural Science Foundation, China (\#ZR2015CL023) and Shandong Province Higher Educational Science and Technology Program (J16LL54). Lin Wang was funded by Weifang City Science \& Technology Project (2015GX019).

\section{Availability of data and materials}

The datasets used and/or analyzed during the current study are available from the corresponding author on reasonable request.

\section{Authors' contributions}

LW and XX performed analysis of the WGS data. YHS and YLS collected samples from the patient, isolated DNA. LM performed STR genotyping. XX drafted the paper. All authors read and approved the final manuscript.

\section{Ethics approval and consent to participate}

The study received ethics approval from the Commission for Scientific Research in Weifang Medical University and was administered in accordance with the ethical standards of the Declaration of Helsinki, second revision. Informed consent was obtained from the patient and his healthy sibling included in the study (ethical approval reference number: WYFY2015-029).

\section{Consent for publication}

Written informed consent was obtained from the patient and his healthy sibling for the publication.

\section{Competing interests}

The authors declare that they have no competing interests.

\section{Publisher's Note}

Springer Nature remains neutral with regard to jurisdictional claims in published maps and institutional affiliations.

\section{Author details}

${ }^{1}$ The School of Physics and Optoelectronic Engineering, Weifang University, Weifang 261061, Shandong, China. ' Laboratory of Clinical Laboratory Diagnostics, Weifang Medical University, Weifang 261053, Shandong, China. ${ }^{3}$ Department of Hematology, Weifang People's Hospital, Weifang 261053, Shandong, China. ${ }^{4}$ Department of Internal Medicine, Florida Hospital, Orlando, Florida 32803, USA. ${ }^{5}$ Stem Cell Lab of the Affiliated Hospital of Weifang Medical University, Weifang 261053, Shandong, China. ${ }^{6}$ College of
Bioscience and Technology, Weifang Medical University, \#1 Building Room 610, 288 Shenglidong Street, Weifang, Shandong Province 261042, People's Republic of China.

Received: 18 August 2017 Accepted: 17 April 2018

Published online: 23 April 2018

\section{References}

1. Arber DA, Orazi A, Hasserjian R, Borowitz MJ, Le Beau MM, Bloomfield CD, et al. The 2016 revision to the World Health Organization classification of myeloid neoplasms and acute leukemia. Blood. 2016;127:2391-406.

2. Meyer C, Hofmann J, Burmeister T, Gröger D, Park TS, Emerenciano M, et al. The MLL recombinome of acute leukemias in 2013. Leukemia. 2013;27: 2165-76. Available from: http://www.pubmedcentral.nih.gov/articlerender. fcgi?artid=3826032\&tool=pmcentrez\&rendertype=abstract.

3. Ren S, Peng Z, Mao J-H, Yu Y, Yin C, Gao X, et al. RNA-seq analysis of prostate cancer in the Chinese population identifies recurrent gene fusions, cancer-associated long noncoding RNAs and aberrant alternative splicings. Cell Res. 2012;22:806-21. Available from: http://www.nature.com/articles/ cr201230?WT.ec_id=CR-201205. http://www.pubmedcentral.nih.gov/ articlerender.fcgi? artid=3343650\&tool=pmcentrez\&rendertype=abstract.

4. Chen Y, Wang L, Xu H, Liu X, Zhao Y. Exome capture sequencing reveals new insights into hepatitis $B$ virus-induced hepatocellular carcinoma at the early stage of tumorigenesis. Oncol Rep. 2013;30:1906-12.

5. Cui J, Yin Y, Ma Q, Wang G, Olman V, Zhang Y, et al. Comprehensive characterization of the genomic alterations in human gastric cancer. Int $J$ Cancer. 2015;137:86-95. Available from: https://onlinelibrary.wiley.com/doi/ abs/10.1002/ijc.29352.

6. Lando M, Fjeldbo CS, Wilting SM, Snoek BC, Aarnes EK, Forsberg MF, et al. Interplay between promoter methylation and chromosomal loss in gene silencing at 3p11-p14 in cervical cancer. Epigenetics. 2015;10:970-80.

7. Zha Y, Marks R, Ho AW, Peterson AC, Janardhan S, Brown I, et al. T cell anergy is reversed by active Ras and is regulated by diacylglycerol kinase-a. Nat Immunol. 2006;7:1166-73.

8. Purow B. Molecular pathways: targeting diacylglycerol kinase alpha in cancer. Clin Cancer Res. 2015;21:5008-12.

9. Batista EL, Warbington M, Badwey JA, Van Dyke TE. Differentiation of HL-60 cells to granulocytes involves regulation of select diacylglycerol kinases (DGKs). J Cell Biochem. 2005:94:774-93.

10. Poli A, Fiume R, Baldanzi G, Capello D, Ratti S, Gesi M, et al. Nuclear localization of diacylglycerol kinase alpha in K562 cells is involved in cell cycle progression. J Cell Physiol. 2017;232:2550-7.

11. Merino E, Sanjuán M a, Moraga I, Ciprés A, Mérida I. Role of the diacylglycerol kinase alpha-conserved domains in membrane targeting in intact T cells. J Biol Chem. 2007;282:35396-404. Available from: http://www. ncbi.n/m.nih.gov/pubmed/17911109.

12. Planey SL, Keay SK, Zhang C-O, Zacharias DA. Palmitoylation of cytoskeleton associated protein 4 by $\mathrm{DHHC} 2$ regulates antiproliferative factor-mediated signaling. Mol Biol Cell. 2009;20:1454-63. Available from: https://www.ncbi. nlm.nih.gov/pmc/articles/PMC2649263/. http://www.molbiolcell.org/cgi/doi/ 10.1091/mbc.E08-08-0849.

13. Yan S-M, Tang J-J, Huang C-Y, Xi S-Y, Huang M-Y, Liang J-Z, et al. Reduced expression of ZDHHC2 is associated with lymph node metastasis and poor prognosis in gastric adenocarcinoma. PLoS One. 2013;8:e56366. Available from: http://www.pubmedcentral.nih.gov/articlerender.fcgi?artid=3574152\&tool= pmcentrez\&rendertype $=$ abstract

14. Li SX, Tang GS, Zhou DX, Pan YF, Tan YX, Zhang J, et al. Prognostic significance of cytoskeleton-associated membrane protein 4 and its palmitoyl acyltransferase DHHC2 in hepatocellular carcinoma. Cancer. 2014;120:1520-31. 